\title{
Nous ne vivons pas dans le meilleur des mondes, mais certainement pas dans le pire non plus
}

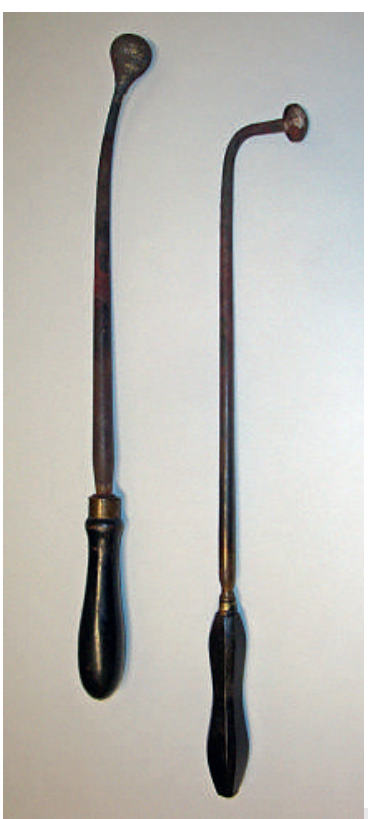

De nos jours, le fer chaud (1800 environ, musée et institut d'histoire de la médecine de l'Université de Zurich) n'est assurément plus l'instrument de choix pour traiter une luxation de la hanche.
En fouinant dans la collection de livres du Musée d'histoire de la médecine de Zurich, je suis tombé sur d'anciens rapports de cas qui m'ont laissé songeur.

Dans une brochure de Robert Henri von Muralt, «Ärzte aus dem Zürcher Patriziat» (Médecins du patriciat de Zurich), on peut lire:

«Le 10 mars 1825, un enfant de 14 ans a été admis à l'hôpital avec une luxation spontanée de la cuisse gauche, la luxation était ascendant car la jambe considérablement était plus courte que l'autre, et comme on notait plusieurs traces de vésicatoires posés antérieurement, décision a été prise d'utiliser le fer chaud.»

J'épargne au lecteur la description précise des «traits de 4-5 pouces de longueur dans la région du trochanter et du cotyle» et me sens heureux que nous puissions compter aujourd'hui sur un repositionnement efficace avec une bonne prise en charge anesthésique et analgésique.

Ailleurs dans le même ouvrage, on trouve le passage suivant:

«Un jour qu'il chevauchait vers Münden, Muralt tomba sur une voiture accidentée dont un cheval de trait s'était échappé. A environ mille pas, il retrouva le valet gisant sur la route avec une jambe cassée.» On ne peut guère reprocher aux passants qui l'entouraient de ne pas savoir que faire pour aider le malheureux, mais quand Muralt demanda de quoi improviser une fixation et un rembourrage pour sa jambe, leur réaction fut négative: «Je leur demandais en effet des mouchoirs ou des foulards, et quand bien même le maître leur assurait: ‘Tout vous sera payé!), ils n'avaient à la fois ni confiance en ses dires, ni assez pitié du valet souffrant pour fournir ce qui leur était demandé en nombre suffisant. Il faut dire que l'exemple du maître n'était pas de nature à inciter les paysans à l'aider, car celui-ci, qui ne cessait de maugréer, n'a donné ni son mouchoir ni son foulard, ne m'a même pas prêté main-forte et m'a à peine remercié.»

Aujourd'hui, il nous paraît aller de soi que la victime d'un accident soit rapidement secourue et prise en charge par des mains compétentes sans égard à sa condition sociale. Dans la grande majorité des cas, l'assistance des professionnels lui est d'ailleurs assurée; c'est l'aide du public présent qui, même de nos jours, peut encore poser problème.

Dans une thèse de doctorat (Sebastian Brändli: «Die Retter der leidenden Menschheit [Les sauveurs de l'humanité souffrante]», 1990), on trouve des passages éloquents sur $l^{\prime}$ «allocation des ressources» voici plus de 200 ans:
«Dans la pharmacopée du jeune Grafferus (1754) figurent, par exemple, deux sortes d'onguents pour hernies: un premier onguent noble et exquis pour gens riches», et un deuxième, «ordinaire, pour les pauvres»».

Loin d'être la cible des détracteurs comme aujourd'hui, la médecine à deux vitesses était visiblement la norme à l'époque. Dans quelle mesure l'onguent «noble et exquis» était plus efficace pour soigner les hernies que l'onguent «ordinaire» est une autre question. La médecine factuelle (evidence based) ne faisait apparemment pas encore partie du plan d'études du «collegium anatomicum».

Même si les caisses-maladie n'existaient pas vers la fin du XVIII siècle, il y avait tout de même des tarifs imposés par les autorités qui tenaient compte de la capacité économique des patients et prévoyaient des remises forfaitaires:

«Pour l'accouchement de l'épouse d'un membre (présumé aisé) de la Constaffel, la sage-femme percevait 10 schillings, 5 schillings pour accoucher l'épouse d'un membre de la société de Schneggen et 3 à 4 schillings chez toutes les autres femmes, 5 pour le premier enfant.»

Indemniser les médecins par des forfaits annuels semble aussi avoir été une pratique courante au dixhuitième siècle: "Mentionnons encore une forme particulière d'honoraire de médecin que l'on trouvait généralement dans les métiers de l'artisanat: le salaire annuel.» On rapporte à ce propos le cas de Konrad Toggenburger, de Marthalen: «Après y avoir exercé son art pendant plusieurs années, il se vit offrir un poste de médecin de ville à Lenzburg (...). Or, quand on sut dans la région qu'il avait l'intention de l'accepter, la perspective de perdre un médecin talentueux et consciencieux suscita une telle crainte que les châtelains et notables des alentours eurent tôt fait de se réunir pour fixer au médecin un salaire annuel, et c'est ainsi qu'ils purent le retenir chez eux, à la grande joie de toute la région.»

Aussi inédits, multiples et difficiles à résoudre que puissent être les problèmes de notre système de santé actuel, on devrait imposer la lecture de ces anciens rapports à tout journaliste ou politicien qui se plaint haut et fort que les temps n'ont jamais été aussi durs. Même s'il souffre de cataracte avancée, il peut espérer lire cet article grâce à la technique de remplacement du cristallin, alors qu'au «bon vieux temps» regretté par quelques nostalgiques, il aurait dû attendre le passage du perceur de cataracte...

Werner Bauer* 